\title{
Least Expensive Facial Mask Against Corona Virus for Short Airway Procedures Generating Aerosol
}

\author{
Vivek Singh $^{1} \cdot$ Krishna Chaubey $^{1} \cdot{\text { Vedak } \text { Onkar }^{1} \cdot \text { Anupam Mishra }}^{1}[$
}

Received: 8 July 2020/Accepted: 20 October 2020/Published online: 28 October 2020

(C) Association of Otolaryngologists of India 2020

\begin{abstract}
With ongoing Corona-pandemic, the quality of personal protection equipment (PPE) across the globe is creating controversy. This article presents a novel design of a facial mask that seems suitable to deal with short airway procedures protecting the surgeon from aerosol infection. The concept, design advantages and limitations are discussed. In absence of good quality PPEs this is an excellent option to deal with airway emergencies.
\end{abstract}

Keywords N95 mask · PPE · Corona virus · Aerosol

\section{Introduction}

The global scarcity of personal protection equipment (PPE) and N95 masks was a real problem for otolaryngologists, dentists and anaesthesiologists during initial few months of covid era. Despite voluminous production of PPEs in India today, the quality of PPEs seems very important during aerosol generating emergency airway procedures (tracheostomy, intubation, direct laryngoscopy, rigid bronchoscopy extraction of foreign body etc.). A variety of N95 masks are available (at GEM portal) ranging from one rupee to that in thousands. Hence the traditional domestic masks manufactured with a label of N95 vary widely in affectivity. Even the well-known authentic masks such as 3 M 1860s and 3 M 1862 N95 respirators are subject to discussions [1] and such comparisons drive their market. The availability of authentic masks in rural areas is limited

Anupam Mishra

amishra_ent@yahoo.com

1 Department of Otolaryngology and Head and Neck Surgery, King George Medical University, Lucknow, India and the serious patients there are dealt with constrained facilities. Even at times, only the surgical mask is available that places a doctor at a tremendous risk. The standard diagnostic RTPCR test is well known for its sensitivity, but portends a $30 \%$ false positive rate. Hence no environment is $100 \%$ safe and even the best of the mask is not $100 \%$ protective. With such ground realities it is rather very risky to opt for any aerosol related procedures particularly with substandard infrastructure. The government is trying its level best with limited resources but even the good hospitals don't possess a negative-pressure OT and it is not surprising to see the doctors dying of corona, the total number of which has crossed 500. It is well known that a variety of existing masks do not offer sufficient viral protection unless impregnated with copper oxide that makes them a biocidal mask [2]. It is hence important to enhance the protection of the existing masks for our fellow otolaryngologists, anaesthesiologists and dentists that actually work with aerosol. A good quality plastic sheet (air-tight envelope) can be considered impermeable for viruses and hence can be protective if superimposed over a mask. But such use is often restricted owing to suffocation. Hence an ideal design would be to introduce a full plastic protection with excellent visualization and virtually minimal suffocation. Considering the logistics in India a novel design of a least expensive facial mask is presented that offers full protection of face/head/neck from direct aerosol and is especially suited for short term procedures.

\section{Materials and Design}

The materials required are: (1) good quality plastic bag, (2) surgical goggles and (3) slender flexible wooden stick with some tensile strength. The plastic envelope completely 
drapes the surgeons head/neck till upper chest where it may be either tied or left semi-open to allow air entry to avoid suffocation. The goggle is integrated with the plastic envelope and is snugly fitted over the face at nasal bridge area to avoid fogging. A diagrammatic representation is depicted in Fig. 1

\section{Design}

The most critical aspect is to select an appropriate sized goggle that snugly fits over the face especially at nasal bridge with no air space between its frame and skin. Thereafter a window is cut about midway on one side of plastic bag corresponding to the external dimension of the goggle glass. Then the goggle is placed through the gap inside plastic bag and edges of the cut-end of window are approximated inside the side-frame of the goggle where they are adhered using transparent packing tape and plastic-adhesives. Several reinforcements ensure an airtight seal. Just beneath the goggle a slender flexible wooden stick is taped making sure that the pointed ends of the stick are secured by additional tape to avoid them penetrating the plastic bag. The plastic bag is then inverted so as to expose the goggle to outside. The wooden stick with its tensile strength keeps the plastic bag somewhat inflated (and reduces its collapse during inspiration). The lower end of the plastic bag can be well tied over the chest all around to create an airtight compartment and this is suitable for ultra-short procedures. Alternatively it can be left loose (open) on all the sides on the chest to prevent suffocation and this is is better suited for a few extra-minutes procedure. To further reduce the suffocation, it is advisable to wear an ordinary mask over the plastic bag (rather than inside directly on facial skin) making sure that the upper

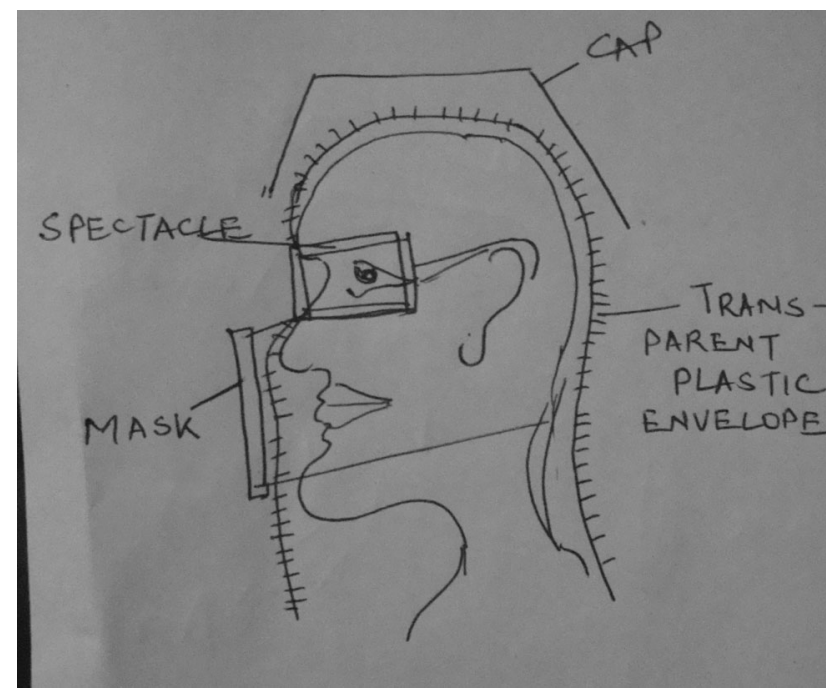

Figure1 Diagrammatic representation of the facial mask knot is tied more tightly to prevent exhaled air escaping superiorly (that causes fogging of glass). More importantly the lower knot of surgical mask is tied very loosely so that if manipulated at chest level it facilitates the air entry like bellows preferably from behind (by an assistant). Figure 2 depicts anterior and the posterolateral views of the facial mask.

Before using this mask should be tested using a domestic scent that is to be copiously sprayed in front on to the goggle with the mask in position. If no smell is appreciated for next $10 \mathrm{~s}$, it is assumed to be airtight. We have used this facial mask even for short 1-15 min otolaryngological procedures such as removal of foreign body from nose, oral/throat examination, tracheostomy, bronchoscopy etc. during this pandemic particularly in absence of PPEs or N95 and with short learning curve (couple of cases) it is recommended under such circumstances. Figure 3 depicts facial mask in position.

This protective design was initially implemented with 16 patients attending the emergency services at the department of otolaryngology where a normal protocol was otherwise followed (Table 1).

\section{Results}

The table depicts the procedure characteristics. All the patients were supposedly CORONA negative by targeted symptoms but not all were subjected to corona testing since the early protocol for testing was particularly targeted for symptomatic cases only. The bellowing was done intermittently in between the procedures when the surgeon complained of suffocation. During bellowing process the surgeon moves away from the patient trolley in a corner of OT. It is worth mentioning that bellowing provided just a temporary relief for a few more minutes only, and on several occasions repeat bellowing was required. In addition a backup fellow resident-surgeon was kept standby in case there was a need for replacing the initial surgeon, but this was never needed. None of our surgeons contacted CORONA infection as evident by their asymptomatic state 5 days after the surgical procedure. It is important to mention that majority of procedures were carried out by the resident-surgeons except for bronchoscopic foreign extraction under general anaesthesia.

\section{Discussion}

This novel design has a great potential of enhancing the protection of our fellow surgeons working with extremely poor resources particularly in rural sectors. It is well known that an exposure of at least $15 \mathrm{~min}$ is necessary to transmit 
Fig. 2 anterior and the posterolateral views of the facial mask. A good quality plastic envelope is desired (transparent plastic sheet is used for better demonstration)
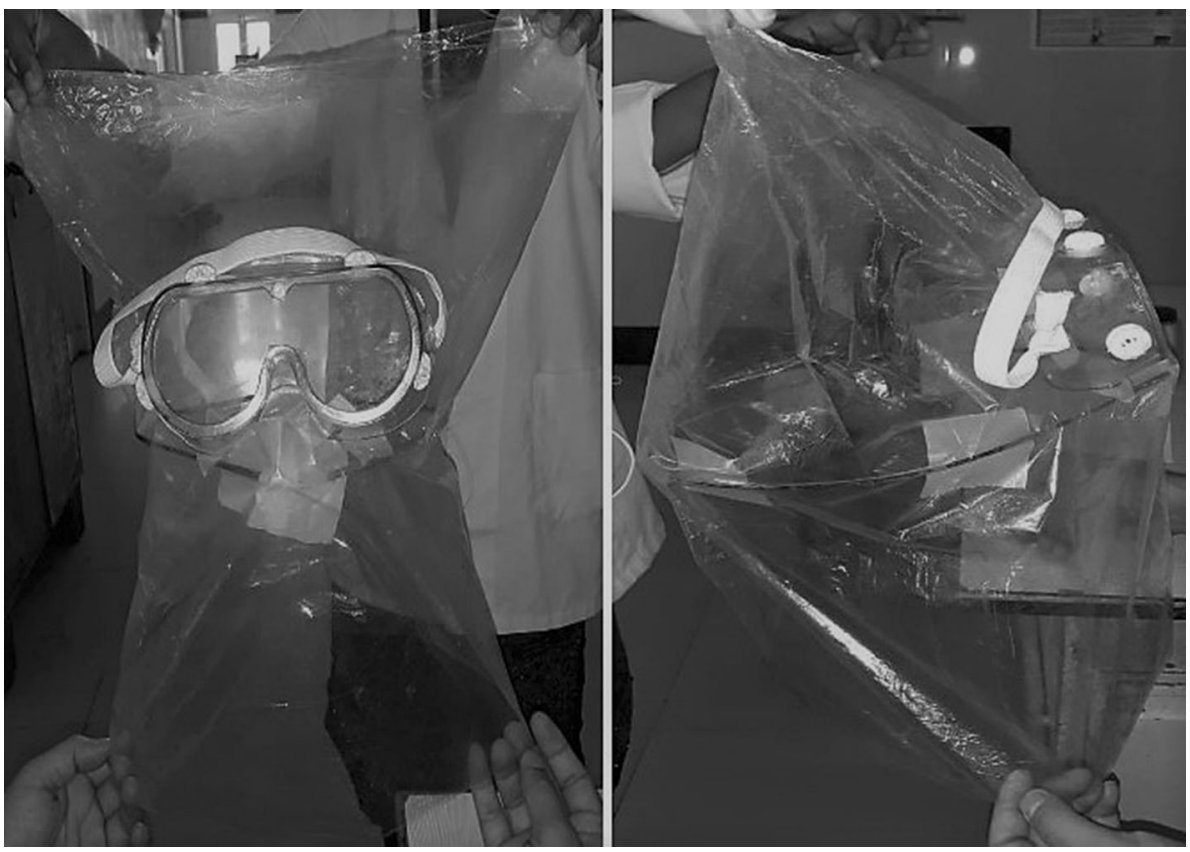

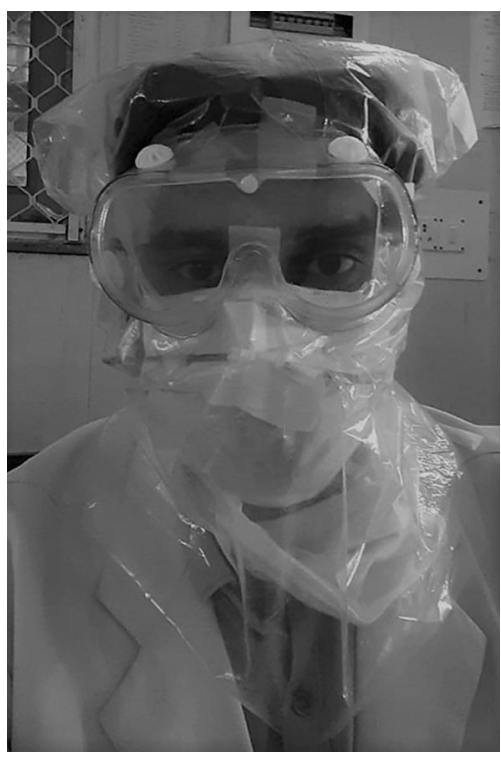

Fig. 3 Facial mask in position. Alternatively the routine surgical mask may be worn outside of the facial mask

the aerosol infection, and wearing such a device will increase this duration to many folds, thereby protecting the physician. The most important aspect is to create an airtight seal especially on the upper (facial) part of this design. The classical 3 methods known to check the affectivity of the mask-seal are user check seal method, qualitative fit testing (QLFT) and quantitative fit testing (QNFT). The United States Occupational Safety and Health Administration recognise both QNFT and QLFT as protocols to determine the fitness of mask. These days, QNFT is considered a gold standard in determining the fit
[3-5]. It includes numerical measurement of the amount of leakage into the mask [5] by an electronic device that measures the ratio of specific particles in the air inside and outside the breathing zone of the mask at the time of it being worn and this ratio directly reflects quantitative leakage. On the other hand QLFT relies on the subjective response of leakage to a test agent (isoamyl acetate or smoke) across nose while wearing the mask [5]. The QLFT may vary with subject's olfaction or be affected by unequal concentration of smell-agents in the air $[6,7]$. Since the user-check-seal method is not applicable in this setting and QNFT was not possible, the QLFT method was used initially using smoke as testing agent but later substituted by an odorant as generating smoke seemed hazardous in hospital atmosphere. Considering the debate on QLFT for assessment of air-seal, if the surgeon is assumed to have normal olfactory function, a latent period of $10 \mathrm{~s}$ seems adequate for the vaporized odour to enter the leakage spot and the use of a pungent smell/scent seems more appropriate (rather than smoke) in such circumstances. We actually sprayed rectified spirit and oldspice/Palmolive after-shave-lotion on to the front of the mask for testing and accordingly we feel that any vaporized scent will solve the purpose. The use of any sensors for olfaction was not feasible in our setup. We did not consider the diameter of virus since our barrier was supposedly impermeable to oxygen molecules (inducing suffocation). Assuming the size of vaporized scent particle to be comparable with smoke we undertook QLFT accordingly.

The required quality of plastic should ideally be absolutely transparent and as thick as possible as a thin plastic may not be able to support the reinforcement-structure 
Table 1 Procedure characteristics

\begin{tabular}{|c|c|c|c|c|}
\hline S no & Surgical procedure & Surgeons time (min) (approx.) & Bellow function/loose enclosure & Tight fit enclosure \\
\hline 1 & Bronchoscopy (foreign body extraction) & $* 4 \min +3 \min +2 \min$ & Twice & No \\
\hline 2 & Endotracheal intubation & $2 \mathrm{~min}$ & No & Yes \\
\hline 3 & Direct laryngoscopy (biopsy) & $3 \mathrm{~min}$ & No & Yes \\
\hline 4 & Direct laryngoscopy (biopsy) & $2 \min$ & No & Yes \\
\hline 5 & Anterior nasal packing & $4 \min$ & No & Yes \\
\hline 6 & Emergency tracheostomy & $* 3 \min +3 \min +3 \min$ & Twice & No \\
\hline 7 & Anterior nasal pack removal & $2 \min$ & No & Yes \\
\hline 8 & Direct laryngoscopy (biopsy) & $3 \min$ & No & Yes \\
\hline 9 & Oesophagoscopy (foreign body extraction) & $* 3 \min +2 \min$ & Once & No \\
\hline 10 & Anterior pack removal & $3 \min$ & No & Yes \\
\hline 11 & Nasal foreign body removal & $1 \mathrm{~min}$ & No & Yes \\
\hline 12 & Bronchoscopy (foreign body extraction) & $* 4 \min +3 \min$ & once & No \\
\hline 13 & Endotracheal intubation & $2 \min$ & No & Yes \\
\hline 14 & Anterior nasal packing & $4 \min +2 \min$ & Once & No \\
\hline 15 & Direct laryngoscopy (biopsy) & $4 \min$ & No & Yes \\
\hline 16 & Direct laryngoscopy (biopsy) & $3 \mathrm{~min}$ & No & Yes \\
\hline
\end{tabular}

*Intermittent bellowing done between the procedure with surgeon away from the trolley. The above numbers are approximate only

inside or give way during bellowing. It is more appropriate to offer conditional bellow function as per the surgeon's requirement, than having persistent holes posteriorly to allow continuous exchange of air inside the bag with outer environment. The breath holding capacity is known to increase with practice as is seen in swimmers. Our experience is best with a 3-4 min procedure, but as the duration increases the bellow-function may be needed. Being introduced for the first time there is no scientific evidence in literature to support this method as being more practical or healthier than examination using a surgical mask with visor. However our limited experience is also not good enough to compare both the modalities but it must be emphasized that considering this dreaded disease (airway surgeons with highest risk of aerosol exposure) this method is equally-practical and may be healthier (at least theoretically) considering the enhanced filtration function of the virus-aerosol.

Limitations (1) Some amount of suffocation is imminent and minimal repeated bellowing of the lower end of bag needs to be done (preferably from behind) as required. (2) Time taken to manufacture this mask may vary from 10 to $15 \mathrm{~min}$ and hence many such masks need to be made daily beforehand and tested with some scent. (3) This is a crude design that is not validated but a real good alternative for working at places without authentic N95 particularly for short emergency airway procedures. (4) It is not recommended for long duration work schedules as this is no substitute for authentic full PPE protection. Furthermore a theoretical possibility of microdroplets does exist in the surroundings and hence duration of exposure with patient and time inside OR needs to be minimum. (5) The plastic sheet needs to be disposed as per the protocol and goggle needs to be recovered with safety.

Advantages (1) Short learning curve. (2) Least expensive as goggle can be reused after soaking in savlon for $2 \mathrm{~h}$. (3) Can be custom prepared by oneself and hence no manufacturing cost. (4) There is no fogging during the procedure since the exhaled air is prevented from entering the goggle because of its snugly fitting frame and the tight approximation of the upper end of the surgical mask literally plastering the plastic sheet tightly on to the skin of midface.

This design should be used by all those health care workers entering the OR dealing with short term aerosol generating procedure particularly when there is no access to full authentic PPE with N95 protection. This design enhances the protection level and hence in airway related emergency procedure there is no better option of selfprotection amidst limited facility. Although we sparingly use this now since we have a satisfactory supply of full PPEs but we still recommend it for those places who are still struggling with the quantity of PPEs or the quality of N95 masks.

Funding The authors are solely responsible for developing their idea through their own funds although the design still needs validation.

\section{Compliance with Ethical Standards}


Conflict of interest The authors declare that they have no conflict of interest.

Ethical Approval All procedures performed in this study were in accordance with the ethical standards of the institutional ethics committee of King George Medical University (KGMU), Lucknow (India), as well as with the 1964 Helsinki declaration and its later amendments or comparable ethical standards.

\section{References}

1. Lam SC, Lee JK, Yau SY, Charm CY (2011) Sensitivity and specificity of the user-seal-check in determining the fit of N95 respirators. J Hosp Infect 77(3):252-256. https://doi.org/10.1016/j.jhin.2010.09.034

2. Borkow G, Zhou SS, Page T, Gabbay J (2010) A novel antiinfluenza copper oxide containing respiratory face mask. PLoS ONE 5(6): $1-8$

3. Tablan OC, Anderson LJ, Besser R, Bridges C, Hajjeh R (2004) $\mathrm{CDC}$ Healthcare infection control practices advisory committee.
Guidelines for preventing health-care-associated pneumonia, 2003: recommendations of CDC and the healthcare infection control practices advisory committee. MMWR Recomm Rep. 26(53)(RR3): $1-36$

4. Centers for Disease Control and Prevention (CDC) (1998) Laboratory performance evaluation of N95 filtering facepiece respirators. MMWR Morb Mortal Wkly Rep 47(48):1045-1049

5. Occupational Safety and Health Administration (2003) Personal protective equipment. US Dep Labor Regul OSHA 64:3151-3212

6. Coffey CC, Lawrence RB, Zhuang Z, Campbell DL, Jensen PA, Myers WR (2002) Comparison of five methods for fit-testing N95 filtering-facepiece respirators. Appl Occup Environ Hyg 17:723-730

7. Marsh JL (1984) Evaluation of irritant smoke qualitative fitting test for respirators. Am Indust Hyg Assoc J 45:245-249

Publisher's Note Springer Nature remains neutral with regard to jurisdictional claims in published maps and institutional affiliations. 